\section{Mortalidade por acidentes de trabalho entre trabalhadores da agropecuária no Brasil, 2000-2010}

\author{
Mortality from work-related accidents among \\ agricultural workers in Brazil, 2000-2010
}

\author{
Mortalidad por accidentes de trabajo entre \\ trabajadores del sector agropecuario en \\ Brasil, 2000-2010
}

Flávia Nogueira Ferreira-de-Sousa ${ }^{1}$ Vilma Sousa Santana 1

\section{Resumo}

Estima-se a mortalidade anual por acidentes de trabalho na agropecuária, 2000-2010, no Brasil. Empregou-se o Sistema de Informações sobre Mortalidade (SIM), para identificar os casos. Dados faltantes para ocupação e acidente de trabalho foram recuperados com informações individuais disponíveis, e incorporados casos ao total. Dados da população são dos Censos Demográficos. Foram identificados 8.923 óbitos por acidentes de trabalho, sendo 44,8\% destes resultantes da recuperação. Em 2000, a mortalidade bruta por acidentes de trabalho foi estimada em 6,4/100 mil trabalhadores, aumentando para 8,1/100 mil em 2003, quando declinou para 7,3/100 mil em 2010. Entre os homens, "projeções de animais ou veículos de tração animal" foram as circunstâncias mais comuns, mas em mulheres foram as intoxicações por agrotóxicos. A mortalidade geral por acidentes de trabalho na agropecuária foi baixa quando comparada à de outros países, sugerindo sub-registro residual apesar da recuperação, o que requer melhoria da qualidade dos registros. Medidas de prevenção sensíveis a diferenças de gênero são necessárias.

Trabalhadores Rurais; Mortalidade Ocupacional; Acidentes de Trabalho; Traumatismos Ocupacionais; Zona Rural

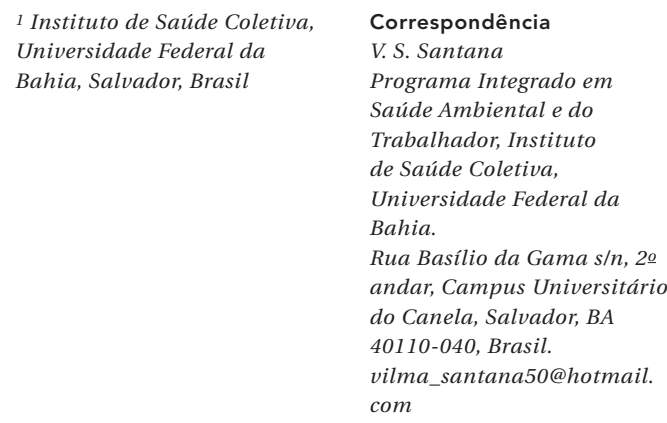




\section{Introdução}

O trabalho na agropecuária compreende atividades na agricultura, pecuária, pesca, aquicultura e silvicultura, e é considerado como de alto risco para acidentes de trabalho em todo o mundo. Nos Estados Unidos, em 2013, a taxa de mortalidade por acidentes de trabalho na agropecuária foi a maior do país, de 22,2 por 100 mil FTE (fulltime equivalent corresponde a trabalhadores em tempo integral) 1 , muito embora menor do que a estimada na Coreia do Sul (30,6 por 100 mil pessoas/ano) em $2012^{2}$. Na Costa Rica, entre 2005 e 2006, essa taxa foi estimada em 11,6 por $100 \mathrm{mil}$ pessoas/ano ${ }^{3}$, próxima a de Taiwan (12,0 por 100 mil pessoas/ano) para o período de 1994-2005 4. No entanto, na União Europeia, em 2010, o coeficiente de mortalidade por acidentes de trabalho na agropecuária foi de apenas 4,5/100 mil trabalhadores, o quinto maior da região 5 .

A tendência do risco de morte por acidentes de trabalho na agropecuária vem sendo de queda, semelhante à observada em trabalhadores de todos os ramos de atividade econômica. Por exemplo, nos Estados Unidos, a taxa de mortalidade por acidentes de trabalho caiu $28,8 \%$ de 20036 a 20131 , média de queda anual de 2,9\%. Tendência de redução dessa mortalidade também foi encontrada em Taiwan ${ }^{4}$ e na Rússia 7 . Dentre os poucos fatores associados aos acidentes de trabalho fatais conhecidos nessa atividade econômica, destaca-se o sexo, cuja razão de masculinidade para a mortalidade foi estimada em 4,2 na Coreia do Sul 2, menor que as relatadas na Costa Rica $(7,6) 3$ e nos Estados Unidos $(11,0) 8$. A idade está associada positivamente com acidentes de trabalho fatais na agropecuária, com aumento linear 2,3,9 ou não linear 7. Achados sobre as circunstâncias envolvidas mostram que o uso de veículos 3,8 , a exemplo de tratores, máquinas e equipamentos agrícolas 2,9,10, animais 10 , bem como quedas de grandes alturas 3,9 , dentre outras, foram as mais comumente observadas, principalmente entre os homens. Entre as mulheres, prevaleceram as situações relacionadas ao transporte, quedas e manuseio de máquinas 2 .

O Brasil é uma das maiores fronteiras agrícolas do mundo, atividade sustentada pelo trabalho de cerca de 12.258.008 pessoas, que representam $14,2 \%$ da população economicamente ativa ocupada, do país, segundo o Censo Demográfico de 2010 11. O Ministério do Trabalho e Emprego reconhece a agropecuária como de alto "risco" para a segurança e saúde dos trabalhadores, classificando-a, e a todos os seus sub-ramos, no grau 3, em uma escala de 1 a 4, à exceção da silvicultura a qual se atribui grau $4{ }^{12}$. Apesar desse alerta para a Saúde Pública, não foram encontrados estudos específicos sobre a mortalidade e/ou fatores associados a acidentes de trabalho para todos os trabalhadores da agropecuária do Brasil. Estudos restritos aos segurados pelo Instituto Nacional do Seguro Social mostram oscilações ao longo do tempo. Por exemplo, em 2003, o coeficiente de mortalidade por acidentes de trabalho nesse ramo econômico foi estimado em 8,5/100 mil, o quarto maior do país 13, aumentando em 2006 para 14,6/100 mil, mas caindo em 2008 para 11,7/100 mil, embora tenha continuado em níveis acima da média nacional geral, de 8,5/100 mil trabalhadores 14. Especificamente para o Estado do Mato Grosso, entre 2002 e 2005, Pignati \& Machado 15 empregaram dados das Comunicações de Acidentes de Trabalho (CAT), da Previdência, para estimar em 58,1/100 mil a mortalidade por acidentes de trabalho na agricultura e pecuária, e em 29,9/100 mil na silvicultura, exploração florestal e pesca. Vale ressaltar que todos esses achados podem estar afetados pelas barreiras no acesso ao reconhecimento e registro dos agravos relacionados ao trabalho, bem como aos benefícios acidentários da Previdência, que se refletem em subestimativas da mortalidade por acidentes de trabalho quando calculadas com dados de sistemas previdenciários. Em uma revisão de estudos, sub-registros de óbitos por acidentes de trabalho nos sistemas de informação da Previdência Social variaram de $39 \%$ a $60,9 \% 13$.

No Brasil, desde 1976, as declarações de óbito passaram a incluir um campo para registro de acidentes de trabalho, a ser preenchido quando a causa básica for do capítulo XX da Classificação Internacional de Doenças, 10a revisão (CID-10), que trata das causas externas. Registros das declarações de óbito estão disponíveis no Sistema de Informações sobre Mortalidade (SIM) do Departamento de Informática do SUS (DATASUS), de amplo acesso, permitindo produzir estimativas para o total dos trabalhadores segurados e não segurados. O SIM é considerado pela Organização Pan-Americana da Saúde e Organização Mundial da Saúde como de boa cobertura, na faixa de $90 \% 16$, e de alta qualidade, com apenas $4,5 \%$ dos óbitos registrados com diagnósticos de causas mal definidas 17. Entretanto, há ainda problemas na qualidade e falta de preenchimento $13,18,19,20$, especialmente nos campos ocupação 19 e acidentes de trabalho 20, que parecem determinar sua pouca utilização no campo da Saúde do Trabalhador. Todavia, na pesquisa epidemiológica é possível a recuperação de informações faltantes empregando-se dados existentes da mesma ou de outras fontes, estratégia cada vez mais utilizada devido à variedade de recursos tecnológicos de computação 
que estão cada vez mais disponíveis 19. Essa recuperação realizada valendo-se em informações do mesmo indivíduo na mesma base de dados 21 pode ser empregada para identificar a relação do acidente com o trabalho, empregando-se registros sobre circunstâncias e local de ocorrência, explícitas nos diagnósticos das causas externas da CID-10, juntamente com dados sobre ocupação, dentre outras porventura existentes.

Neste estudo estima-se o coeficiente de mortalidade por acidentes de trabalho na agropecuária no Brasil, entre 2000 e 2010, descrevendo-o de acordo com o sexo, idade, diagnósticos específicos e evolução histórica no período estudado. Estimativas foram padronizadas por idade. O número total de casos foi estimado acrescentandose aos existentes os dados recuperados.

\section{Métodos}

Este estudo foi conduzido com a base de dados SIM e dados populacionais agregados de trabalhadores obtidos nos Censos Demográficos de 2000 e 2010, do Instituto Brasileiro de Geografia e Estatística (IBGE). A população de referência do estudo foi a população economicamente ativa ocupada, restrita aos que tinham de 18 a 75 anos de idade e trabalhavam na agropecuária. Essa corresponde à Seção A, Divisões 1, 2 e 3 da Classificação Nacional de Atividades Econômicas 2000 (CNAE 2.0) e compreende ocupações da agricultura (códigos 11 a 14), pecuária (15 a 17), produção florestal (21 a 23), pesca e aquicultura (31 e 32). Como a informação sobre a CNAE inexiste na declaração de óbito, os casos da agropecuária foram identificados com base na ocupação, especificamente, os registrados como da Família 6 da Classificação Brasileira de Ocupações (CBO) versão 2002.

Do SIM foram extraídas planilhas contendo dados individuais sociodemográficos, como a data do nascimento, sexo, diagnósticos das causas básicas e associadas, bem como a data do óbito e os registros dos campos específicos para ocupação habitual e acidentes de trabalho. Do IBGE extraíram-se dados da população economicamente ativa ocupada, empregando-se o sistema SIDRA, para cada um dos anos censitários. Dados intercensitários foram estimados por interpolação, assumindo-se variação linear no intervalo de tempo considerado. Como o número de trabalhadores, por sexo e grupo de idade, não estava disponível para cada ramo de atividade econômica, estes dados foram estimados para a agropecuária utilizando-se pesos, que correspondem às proporções respectivas da população economicamente ativa ocupada total.
As variáveis do estudo foram criadas baseando-se nas originais do SIM, especificamente: Var1 - "ocupação na agropecuária” que corresponde à Família 6 da CBO (sim, não, ignorado); Var2 - "grupo diagnóstico causas externas" correspondendo ao capítulo XX da CID-10 (sim, não); Var3 - "campo acidente de trabalho" (sim, não, ignorado); Var4 - "subgrupo diagnóstico das causas externas que especifica 'fazenda' como local de ocorrência do acidente” (sim, não). Essas variáveis foram empregadas para a identificação dos casos totais de acidentes de trabalho na agropecuária, recuperando-se os dados faltantes de cada uma delas com informações disponíveis das demais, complementarmente. Em resumo, casos do estudo são óbitos cuja ocupação era agropecuária (Var1 = sim), o grupo diagnóstico era causa externa (Var2 = sim), tinha reconhecimento no campo acidentes de trabalho (Var3 $=$ sim), conformando a trilha diagnóstica 1. Adicionalmente, criou-se a trilha diagnóstica 2, valendo-se dos casos que eram da agropecuária (Var1 $=$ sim), haviam falecido por causas externas (Var2 $=$ sim), tinham o campo acidente de trabalho com registro ignorado (Var3 = ignorado), mas o subgrupo diagnóstico da CID-10 referia ocorrência na "fazenda" (Var4 = sim). Por último, empregou-se a trilha diagnóstica 3, para os casos que não tinham a ocupação registrada (Var1 = ignorado), mas o diagnóstico era causa externa (Var2 $=\operatorname{sim}$ ), era reconhecido como acidente de trabalho no campo acidente de trabalho (Var3 = sim) e o CID-10 referia "fazenda" como o local do acidente (Var4 = sim). Portanto, a recuperação de casos baseou-se em dados de variáveis existentes na própria base, do mesmo indivíduo, empregando-se as deduções lógicas explicitadas. O total de acidentes de trabalho resultou do somatório dos casos identificados nas trilhas 1, 2 e 3. Não foram incluídos suicídios, embora possam estar relacionados ao trabalho da agropecuária 22 . Variáveis descritoras foram sexo, grupo de idade em anos (18-24, 25-44, 4049, 45-59, e 60-75 anos), ano do óbito e os códigos específicos de quatro dígitos da CID-10.

Para analisar a qualidade do preenchimento da declaração de óbito e estimar o sub-registro correspondente a cada uma das informações, estimou-se a proporção de dados faltantes das Var1, Var2, e Var3. Para avaliar a qualidade do preenchimento da CID-10 utilizou-se a proporção de diagnósticos de causas mal definidas, que correspondem ao capítulo XVIII da CID-10 (R00 a R99). Os coeficientes de mortalidade anuais por acidentes de trabalho na agropecuária foram calculados dividindo-se o número de casos destes acidentes pela população de trabalhadores correspondente, para o número de casos total, 
compreendendo os existentes e recuperados, e separadamente apenas os existentes. O coeficiente de mortalidade padronizado por idade foi calculado empregando-se o método direto, tendo como população padrão o total dos trabalhadores da agropecuária do Censo Demográfico de 2010. A análise foi realizada com o SAS 9.2 (SAS Inst., Cary, Estados Unidos) e planilhas Excel (Microsoft Corp., Estados Unidos). O projeto foi registrado no Sistema Nacional de Ética em Pesquisa, e aprovado pelo Comitê de Ética em Pesquisa do Instituto de Saúde Coletiva da Universidade Federal da Bahia (protocolo no135.771, 30/Out/2012). Os dados empregados foram anônimos, e os resultados, apresentados de modo agregado.

\section{Resultados}

Foram encontrados 6.667 .331 óbitos registrados no período entre 2000 e 2010 no SIM, excluídos os casos de suicídio (Figura 1). Desses, 2.287.128 $(34,3 \%)$ não contavam com o registro da ocupação, e 841.526 (12,6\%) estavam identificados com ocupação na agropecuária, dos quais 165.037 (19,6\%) tinham como causa básica ou associada da morte, causas externas. Todavia, para esses últimos casos o campo acidente de trabalho estava preenchido com sim ou não, apenas para 42.423 (25,7\%), sendo que destes, 4.930 (3\%) foram identificados e registrados como acidentes de trabalho (trilha diagnóstica 1). Empregandose a trilha diagnóstica 2 recuperaram-se 3.586 casos, e com a de número 3, 407 casos de acidentes de trabalho na agropecuária. Assim, totalizaram 8.923 casos de interesse para o estudo, sendo $44,7 \%$ ( $n=3.993$ ) deles recuperados com base nas informações disponíveis no SIM, 40,2\% com a trilha diagnóstica 2 e 4,6\% com a trilha 3 .

Na Tabela 1, apresentam-se dados sobre a evolução da qualidade do preenchimento da declaração de óbito em relação a acidentes de trabalho. Verifica-se que no início do estudo, em 2000, o campo ocupação não era preenchido com registros válidos em pouco mais da metade das declarações de óbito (50,3\%). Isso reduziu até 2005, ano em que passou a aumentar até 2010. Em todo o período do estudo houve uma variação de $+56,2 \%$. O campo acidentes de trabalho, cujo preenchimento é requerido para todos os óbitos por causas externas, não continha registros válidos na maioria das declarações. Em todos os anos, a proporção de não preenchimento ficou acima de $80 \%$ em todos os grupos ocupacionais, mas um pouco menor na agropecuária, embora no patamar de $70 \%$. Com os dados da Tabela 1 verifica-se também que, em 2006, es- timaram-se as maiores proporções de registros ignorados/ausentes no campo ocupação (65,9\%) e acidente de trabalho $(86,1 \%)$ para todas as ocupações, e para a agropecuária separadamente $(80,2 \%)$. Ainda nessa Tabela 1, observa-se que a porcentagem de causas mal definidas teve tendência de queda nessa década, caindo de 11,1\% para $5,5 \%$, redução de $50,5 \%$ e de $65,3 \%$ para todos os grupos ocupacionais e na agropecuária, respectivamente (Tabela 1 ).

Os coeficientes brutos de mortalidade por acidentes de trabalho estimados com o número de casos totais, existentes e recuperados, foram 6,4/100 mil trabalhadores em 2000 e 7,3/100 mil em 2010, o que corresponde a um aumento de $14,1 \%$ em todo o período do estudo (Figura 2). Entretanto, inicialmente esse coeficiente aumentou atingindo o pico de 8,1/100 mil em 2003, quando passou a reduzir até 2006, atingindo o seu menor nível, igual ao inicial, de 6,4/100 mil. A partir de 2006, voltou a elevar até 2008 alcançando 7,8/100 mil, quando passou a diminuir até o final do estudo em 2010. A distribuição das estimativas padronizadas por idade foi muito próxima à observada com as medidas brutas. Ainda na Figura 2, observa-se que os coeficientes estimados com dados dos casos existentes ficaram abaixo das estimativas brutas e padronizadas por idade, calculadas com o total dos casos, em todos os anos estudados. As variações da distribuição dessas medidas foram próximas às encontradas para as demais.

Na Figura 3, verifica-se que a elevação do coeficiente bruto de mortalidade por acidentes de trabalho, no período, ocorreu para ambos os sexos, mas foi bem menor entre os homens $(15,3 \%)$ quando comparados às mulheres (27,3\%). Entretanto, os coeficientes de mortalidade por acidentes de trabalho para os homens foram sempre maiores que os estimados para as mulheres, com razão bruta de mortalidade homem/mulher em 2010 de 7\%. Não foi possível o ajustamento por idade para as medidas específicas por sexo, devido aos pequenos números de casos em mulheres. Houve também aumento da mortalidade por acidentes de trabalho com a faixa de idade, em todos os anos do estudo; a tendência entre 2000 e 2010 foi de crescimento da mortalidade apenas para trabalhadores acima de 40 anos, e de aumento até 2002 e 2003 para os jovens de 18-24 e 25-39 anos, respectivamente, com discreta queda até 2010 (Figura 4).

$\mathrm{Na}$ Tabela 2, apresentam-se as distribuições dos diagnósticos das causas básicas de morte mais comuns, entre os acidentes de trabalho na agropecuária, de acordo com o sexo. O diagnóstico mais frequente foi o V80.0 - quedas, ejeções ou projeções de animais ou veículos de tração 


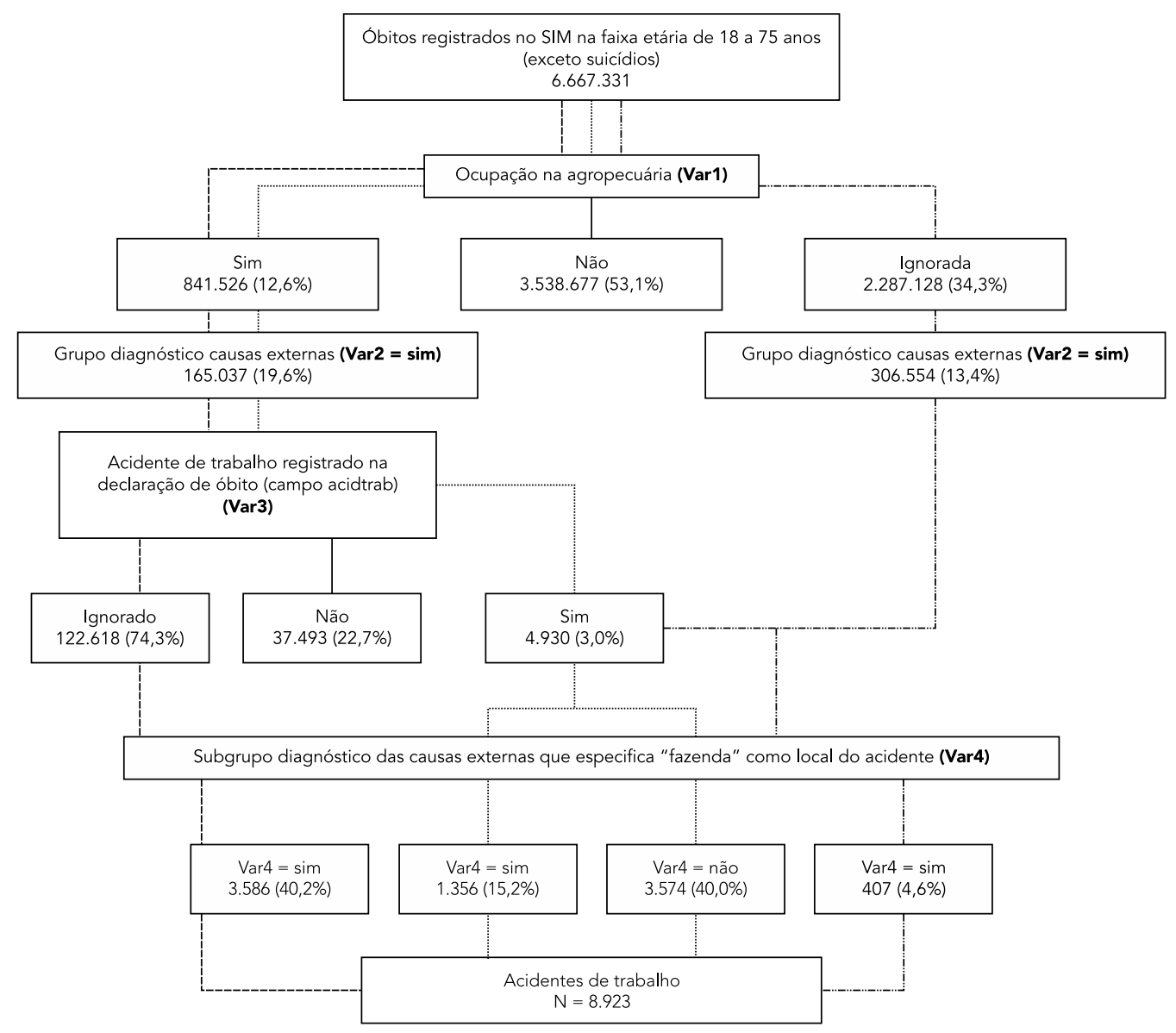

animal com 634 casos (7,1\% do total), seguido pelo V84.9 - acidente envolvendo veículos de motor essencialmente agrícola $(n=455 ; 5,1 \%)$, e em terceiro lugar, o X95.7 - agressões interpessoais com arma de fogo ( $n=428 ; 4,8 \%)$. Essa ordem se repete entre os homens, mas difere do encontrado para as mulheres, cujo diagnóstico da causa básica de óbito por acidente de trabalho mais referido foi Y18.9 - intoxicações por agrotóxicos ( $\mathrm{n}=30 ; 7,5 \%)$, seguido por V80.0 ( $\mathrm{n}=$ $21 ; 5,3 \%)$, e V84.9 ( $n=16 ; 4 \%)$, respectivamente. Nenhum óbito obteve o diagnóstico codificado com as causas externas relacionadas com o trabalho na CID-10 (Y96, Z04.2, Z56.1 a Z57.9). Vale notar que, entre as 16 causas mais comuns de morte por acidentes de trabalho, destacaram-se também as agressões por instrumento cortante (X99.7) e contundente (Y00.7) que se concentra- ram no sexo masculino. A eletrocussão também esteve entre as causas imediatas mais comuns dos acidentes de trabalho na agropecuária.

\section{Discussão}

Neste estudo estimou-se que no Brasil, entre 2000 e 2010, 8.923 trabalhadores da agropecuária faleceram em decorrência de acidentes de trabalho. Com esses dados, em 2010, o coeficiente bruto de mortalidade anual estimado foi de 7,3/100 mil trabalhadores, igual à estimativa padronizada por idade. Não foram encontradas diferenças entre estimativas brutas e ajustadas por idade. Durante o período do estudo, a distribuição dos coeficientes brutos formou duas curvas, com pico de 8,1/100 mil em 2003, e outro de 7,8/100 mil 
Tabela 1

Indicadores da qualidade do registro dos dados da declaração de óbito em todos os grupos ocupacionais e na agropecuária, por ano do óbito. Brasil, 2000-2010.

\begin{tabular}{|c|c|c|c|c|c|}
\hline \multirow[t]{2}{*}{ Ano do óbito } & \multirow[t]{2}{*}{ Registro do campo ocupação * (\%) } & \multicolumn{2}{|c|}{ Registro do campo acidente de trabalho ** } & \multicolumn{2}{|c|}{ 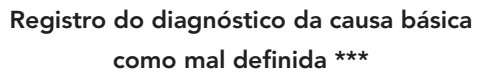 } \\
\hline & & $\begin{array}{l}\text { Todos os grupos } \\
\text { ocupacionais (\%) }\end{array}$ & $\begin{array}{l}\text { Apenas } \\
\text { agropecuária (\%) }\end{array}$ & $\begin{array}{l}\text { Todos os grupos } \\
\text { ocupacionais (\%) }\end{array}$ & $\begin{array}{l}\text { Apenas } \\
\text { agropecuária (\%) }\end{array}$ \\
\hline 2000 & 50,8 & 83,3 & 76,1 & 11,1 & 19,9 \\
\hline 2001 & 48,4 & 81,8 & 74,4 & 11,0 & 20,0 \\
\hline 2002 & 47,3 & 79,6 & 72,4 & 10,5 & 18,9 \\
\hline 2003 & 47,3 & 78,9 & 71,3 & 10,1 & 18,1 \\
\hline 2004 & 46,7 & 78,1 & 71,0 & 9,6 & 16,9 \\
\hline 2005 & 46,1 & 74,9 & 69,3 & 8,1 & 13,2 \\
\hline 2006 & 65,9 & 86,1 & 80,2 & 6,5 & 8,6 \\
\hline 2007 & 62,3 & 85,2 & 79,2 & 6,1 & 7,7 \\
\hline 2008 & 58,2 & 84,5 & 78,0 & 5,9 & 7,5 \\
\hline 2009 & 57,4 & 83,8 & 76,9 & 5,7 & 7,1 \\
\hline 2010 & 56,2 & 82,1 & 75,5 & 5,5 & 6,9 \\
\hline
\end{tabular}

Fonte: Departamento de Informática do SUS. Sistema de Informações sobre Mortalidade.

* Var1 = ausente e ignorado;

** Var3 = ausente e ignorado;

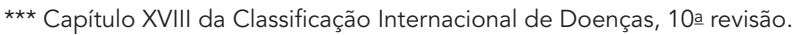

Figura 2

Coeficientes de mortalidade (por 100 mil trabalhadores) por acidentes de trabalho na agropecuária, calculados com dados existentes e recuperados brutos, existentes e recuperados padronizados por idade e existentes, por ano. Brasil, 2000-2010.

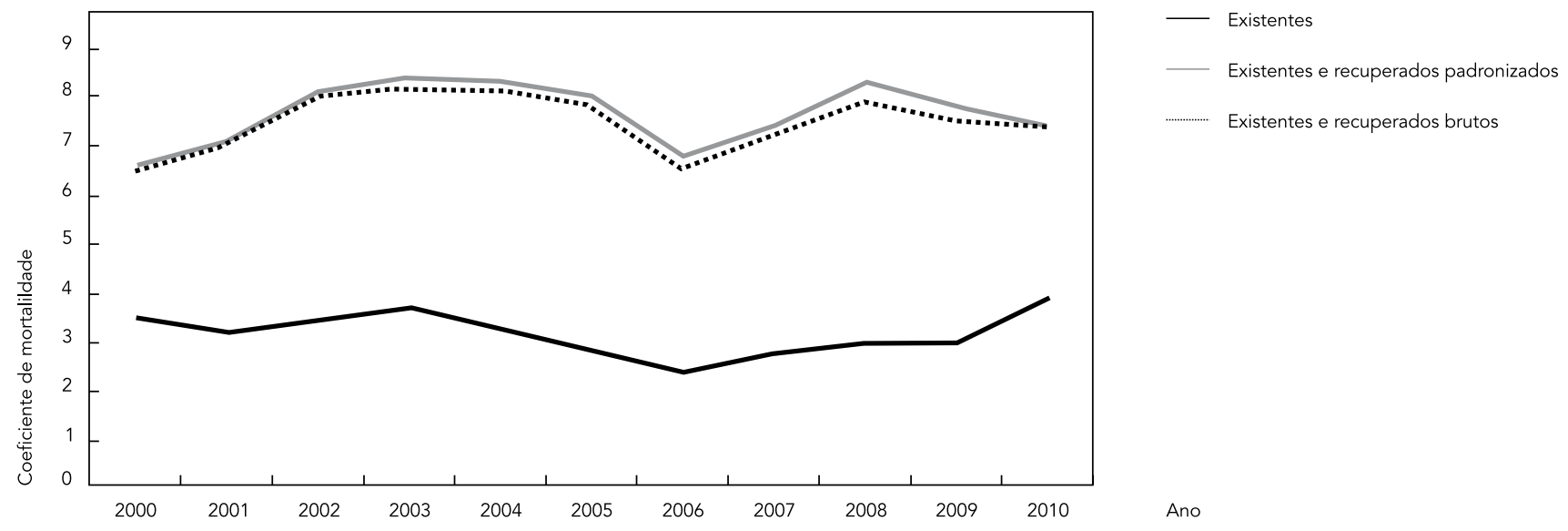


Coeficiente de mortalidade (por 100 mil trabalhadores) por acidente de trabalho na agropecuária, de acordo com o sexo e ano calendário.

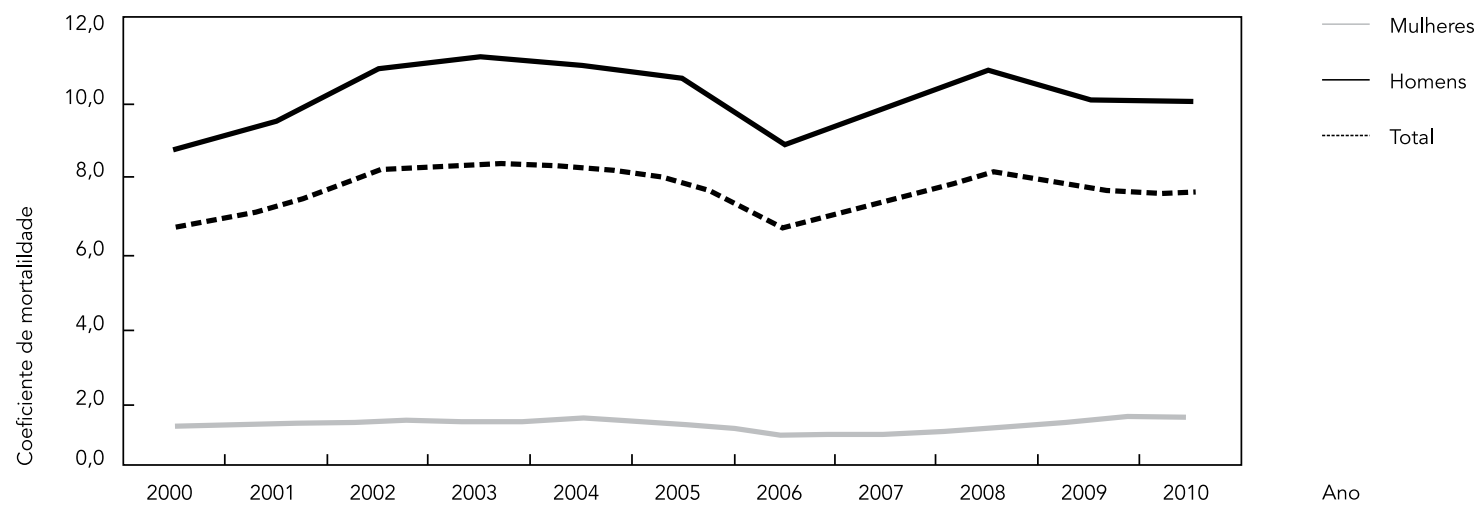

Figura 4

Coeficiente de mortalidade (por 100 mil trabalhadores) por acidentes de trabalho na agropecuária de acordo com faixa de idade. Brasil, $2000-2010$.

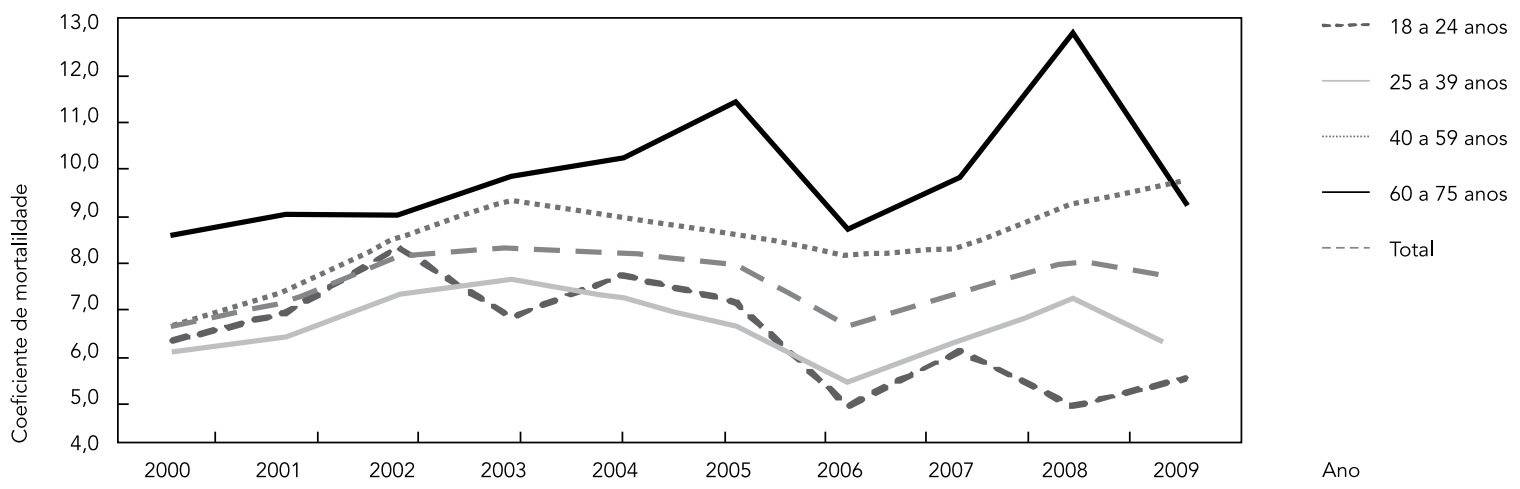

em 2008, ficando os menores valores em $2000 \mathrm{e}$ 2006, iguais, de 6,4/100 mil trabalhadores. No geral, entre 2000 e 2010 a tendência foi de aumento da estimativa, de $14,1 \%$. Embora o número de óbitos por acidentes de trabalho na agropecuária tenha sido expressivo, aumentou consideravelmente após a recuperação de casos não identificados, que representaram $44,7 \%$ do total analisado. Assim, as estimativas com dados totais, existentes e recuperados, ficaram maiores do que as realizadas apenas com dados existentes em todos os anos estudados. Para as estimativas específicas por sexo, homens que trabalhavam na agropecuária tiveram maior risco de morrer por acidente de trabalho do que as mulheres, em todos os anos. Devido aos maiores valores, o sexo masculino apresentou a mesma tendência descrita para a mortalidade por acidentes de trabalho na agropecuária em geral, enquanto entre as mulheres houve pequenas oscilações com discreto aumento para o período, maior do que o encontrado entre os homens. Com relação à idade, o padrão de variação mais comum foi de crescimento da mortalidade entre 2000 até 2002 
Distribuição dos óbitos por acidentes de trabalho na agropecuária por diagnóstico da causa básica (CID-10) e sexo. Brasil 2000-2010.

\begin{tabular}{|c|c|c|c|}
\hline Causa básica do óbito (CID-10 Causas Externas) & $\begin{array}{c}\text { Homens } \\
\text { n (\%) }\end{array}$ & $\begin{array}{c}\text { Mulheres } \\
\text { n (\%) }\end{array}$ & $\begin{array}{l}\text { Total } \\
\text { n (\%) }\end{array}$ \\
\hline 1) Quedas ou ejeção de animais ou veículos de tração animal (V800) & $613(7,2)$ & $21(5,3)$ & $634(7,1)$ \\
\hline 2) Acidente envolvendo veículo de motor essencialmente agrícola (V849) & $439(5,1)$ & $16(4,0)$ & $455(5,1)$ \\
\hline 3) Agressões interpessoais com arma de fogo (X957) & $419(4,9)$ & $9(2,3)$ & $428(4,8)$ \\
\hline 4) Impacto com objetos lançados, projetados ou em queda em fazendas (W207) & $400(4,7)$ & $2(0,5)$ & $402(4,5)$ \\
\hline 5) Pessoa montada em animal ou veículos de tração animal sem especificação (V809) & $300(3,5)$ & $5(1,3)$ & $305(3,4)$ \\
\hline 6) Agressão por meio de objeto cortante (X997) & $263(3,1)$ & $14(3,5)$ & $277(3,1)$ \\
\hline 7) Impacto por objeto lançado em local não especificado (W209) & $247(2,9)$ & $1(0,25)$ & $248(2,8)$ \\
\hline 8) Intoxicações por agrotóxicos (Y189) & $177(2,1)$ & $30(7,5)$ & $207(2,3)$ \\
\hline 9) Condutor de veículo essencialmente agrícola (V845) & $200(2,3)$ & $3(0,8)$ & $203(2,3)$ \\
\hline 10) Exposição a fatores não especificados (X599) & $190(2,2)$ & $4(1,0)$ & $194(2,2)$ \\
\hline 11) Impacto por objeto lançado em outros locais não especificados (W208) & $174(2,0)$ & $2(0,5)$ & $176(2,0)$ \\
\hline 12) Acidente com veículo a motor (V892) & $152(1,8)$ & $5(1,3)$ & $157(1,8)$ \\
\hline 13) Exposição à corrente elétrica em fazendas (W877) & $147(1,7)$ & $9(2,3)$ & $156(1,7)$ \\
\hline 14) Exposição à corrente elétrica em local não especificado (W879) & $102(1,2)$ & $2(0,5)$ & $104(1,2)$ \\
\hline 15) Agressão por objeto contundente em fazendas (Y007) & $100(1,2)$ & $4(1,0)$ & $104(1,2)$ \\
\hline 16) Outros diagnósticos & $4.602(54,0)$ & $271(68,1)$ & $4.843(54,6)$ \\
\hline Total & 8.545 & 398 & 8.923 \\
\hline
\end{tabular}

Fonte: Departamento de Informática do SUS. Sistema de Informações sobre Mortalidade.

CID-10: Classificação Internacional de Doenças, 10ạ revisão.

e 2003, quando as curvas ficaram levemente descendentes até 2010, em todas as faixas, exceto entre os que tinham idades entre 40 e 59 anos, para os quais a tendência foi de discreto aumento. Ademais, verificou-se que os diagnósticos mais comuns foram quedas, ejeções ou projeções de animais ou veículos de tração animal entre os homens, mas as intoxicações por agrotóxicos foram as primeiras causas de morte por acidentes de trabalho em mulheres da agropecuária.

Apesar dos esforços de recuperação de casos de óbitos por acidentes de trabalho na agropecuária, as estimativas de mortalidade deste estudo parecem ainda estar afetadas por sub-registro. Evidências disso são os valores da mortalidade menores do que os de outros países, conhecidos por melhores condições de trabalho, como o Canadá (10,1/100 mil), Itália (11,9/100 mil), Bulgária (15,7/100 mil), Croácia (15,9/100 mil), República Tcheca (13,4/100 mil) e Suécia (14,6/100 mil), bem como outros como Turquia (21/100 mil) e Argentina (30/100 mil) (International Labour Office - ILO. http://laborsta.ilo.org, acessado em 27/Mar/2012). Nossas estimativas, todavia, foram maiores do que a média da França (5,9/100 mil), Portugal (3,9/100 mil) e Espanha (3,8/100 mil) (ILO. http://laborsta.ilo.org, acessado em 27/Mar/2012). Essas comparações estão limitadas porque os achados desses países se referem apenas a trabalhadores segurados e foram utilizados registros de sistemas de informação previdenciários, enquanto que neste estudo a fonte de dados é o SIM, de cobertura universal, para segurados e não segurados. Nossas estimativas foram também menores do que as disponibilizadas pela Previdência Social, para o período entre 2006 e 2008, que passaram de 14,6/100 mil para 11,7/100 mil 14. Essas diferenças revelam que estimativas mais precisas ainda são necessárias porque é pouco plausível que condições de segurança nos ambientes de trabalho da agropecuária sejam melhores do que as das outras atividades econômicas do país 15,22. A agropecuária se desenvolve principalmente em áreas rurais ou pouco povoadas, onde são comuns a precariedade da qualidade, oferta e do acesso a serviços públicos que podem contribuir para a má qualidade dos registros de agravos relacionados ao trabalho. Além disso, é elevada a informalidade do emprego e dos empreendimentos agrícolas, muitos deles familiares, não registrados ou voltados para a agricultura de subsistência, notadamente em áreas rurais pobres 22 . A falta de identificação da relação de mortes por causas externas com o trabalho é menor em trabalhadores informais em comparação com os formais, no 
SIM 18, e no Sistema de Informação de Agravos de Notificação, (SINAN) 14, reforçando a interpretação de sub-registro das estimativas deste estudo para a agropecuária.

Embora entre 2000 e 2010 a tendência da mortalidade por acidentes de trabalho tenha sido de pequeno crescimento, a partir de 2003 pode-se estimar um leve declínio até 2010. Isso contrasta com a queda acentuada observada em outros países, a exemplo dos Estados Unidos 1,6, Taiwan ${ }^{4}$ ou Rússia 7 , que mostraram declínio nas últimas décadas. $\mathrm{E}$ também foi observado para o total de trabalhadores, como nos Estados Unidos 1,6, Austrália 23, Europa e Croácia (ILO. http://laborsta.ilo.org, acessado em 27/Mar/2012), e no Brasil 13,14,24. Em geral, essa diminuição parece ser resultado da maior participação no mercado de trabalho de atividades econômicas de menor risco de morte por acidentes, como os serviços e comércio, bem como a adoção de práticas mais seguras, decorrentes do aprimoramento tecnológico dos processos de trabalho, do melhor nível de educação e qualificação ocupacional dos trabalhadores, e da sua mais eficiente atuação na reivindicação por melhores condições de trabalho. No Brasil, pode-se também mencionar o crescimento da cobertura das ações do SUS e da aplicação de instrumentos de desincentivos pela Previdência. Nesta investigação, todavia, as estimativas parecem evidenciar que há ainda muito a ser feito em relação ao registro de casos e na melhoria das condições de segurança no trabalho agropecuário. De fato, com os dados do SIM, as estimativas de mortalidade por acidentes de trabalho oscilaram com tendência de queda nos últimos sete anos, mesmo com a melhoria da cobertura do SIM e, possivelmente, redução do subregistro dos casos. Entretanto, o aumento tem sido pequeno e a tendência é de estabilização em quase todas as regiões 25 . Ademais, embora tenha havido avanço na especificação dos diagnósticos e redução de códigos relativos a causas mal definidas, isto não vem ocorrendo para o registro no campo da ocupação ou dos acidentes de trabalho, especialmente na agropecuária. Digno de nota é que apesar das dificuldades práticas do registro da ocupação, que requer a consulta de manuais, neste estudo a proporção de dados faltantes foi menor do que a do campo acidentes de trabalho, apesar de ainda oscilar em torno dos $50 \%$, com tendência de elevação a partir de 2005.

Esta pesquisa confirma os achados de estudos de outros países 1,3,6,8 e também do Brasil 14 de que homens têm maiores coeficientes de mortalidade por acidentes de trabalho na agropecuária do que as mulheres, demonstrando que as atividades de trabalho desenvolvidas pelo sexo masculino tendem a ser menos seguras. Entre- tanto, chama a atenção que a primeira causa de morte entre as mulheres tenha sido as intoxicações ocupacionais por agrotóxicos, o que alerta para a necessidade de mais estudos sobre as causas dessa desvantagem. É possível que esses achados revelem menor controle do uso dessas substâncias quando os trabalhadores são mulheres, por exemplo, tendo menor acesso à informação, a empregos com melhores condições de trabalho, ou o crescente envolvimento em atividades menos tradicionais para o sexo feminino na agropecuária. Ou mesmo o aumento da naturalização do trabalho nessa atividade, considerado mais como "ajuda” do que uma ocupação entre as mulheres 26 .

A mortalidade por acidentes de trabalho na agropecuária no Brasil se elevou com a faixa de idade em todos os anos do estudo, com variações mais consistentes a partir de 25 anos de idade. Esse resultado confirma a associação direta com a idade verificada em outros países $2,3,7$, que se assemelha ao observado para os acidentes de trabalho fatais em todos os ramos de atividades econômicas 13,14,25. Em geral, ocupações ou tarefas de maior risco são proibidas para menores, e podem ser menos comumente atribuídas aos jovens nas atividades agrícolas perigosas, mesmo considerando a extensa informalidade nesse ramo de atividade, que o coloca fora do âmbito de cobertura das inspeções formais do Ministério do Trabalho e Emprego. Pessoas com mais idade podem ter comprometimento do equilíbrio, atenção, redução da massa e força muscular, fatores associados à ocorrência de acidentes em geral, como também com os relacionados ao trabalho ${ }^{9}$. Mas outros fatores não pessoais podem ter um papel importante nesse contexto, como as condições de trabalho de idosos na agropecuária, o que requer estudos específicos sobre esta temática. A redução da mortalidade por acidentes de trabalho entre os mais jovens, a partir de 2002, pode ser consequência das ações efetivas de combate ao trabalho infantil no país e melhoria das condições de trabalho de adolescentes 25 .

Notar que embora a mecanização da agricultura, com o uso intensivo de implementos agrícolas, pareça ter um papel importante para as mortes por acidentes de trabalho na agropecuária 7,9,27,28, nesta pesquisa a maior parte dos casos envolveu o transporte animal, em montaria ou com veículos de tração animal, o que implica recomendações especiais para a prevenção deste tipo de acidente. Não obstante, deve-se destacar o aumento da venda de tratores e máquinas agrícolas no mercado interno brasileiro, que cresceu 53,4\% entre 2002 e 2011, e de agrotóxicos que aumentou 89\% de 2000 a 2009 (Ministério de Agricultura, Pecuária e Abastecimento. 
http://www.agricultura.gov.br/, acessado em 07/Dez/2014), estes últimos já mencionados como a principal causa de acidentes de trabalho em mulheres. O perfil das circunstâncias dos acidentes de trabalho fatais observado foi diferente do encontrado na Índia, onde $77,6 \%$ dos acidentes de trabalho na agropecuária se relacionavam a máquinas agrícolas 27 , enquanto que nos Estados Unidos, 43\% dos acidentes na agropecuária envolveram tratores agrícolas 28 .

Os achados deste estudo também revelaram uma melhoria da qualidade do diagnóstico da causa básica ao longo do período, evidente na redução dos casos classificados como causas mal definidas. Isso não ocorreu para o preenchimento do campo acidentes de trabalho, e ao contrário, houve uma pequena piora para o registro do campo ocupação. Vale notar que, os menores valores do coeficiente de mortalidade por acidentes de trabalho estimados em 2006 coincidem com as maiores estimativas de registros de ausentes/ ignorados para a ocupação e acidentes de trabalho, tanto para o geral como para a agropecuária em particular. Esse resultado pode evidenciar possíveis fatores relacionados ao registro no sistema de informação sobre mortalidade, que à época era alvo de discussões relativas a mudanças implementadas nos anos subsequentes 24 , e não a alterações reais da mortalidade por acidentes de trabalho.

As conclusões desta pesquisa são limitadas devido ao possível sub-registro residual de casos, não superado totalmente com a recuperação, como discutido previamente. Essa estratégia foi conduzida apenas com o SIM, mas poderia empregar registros de outros sistemas de informação universais, que incluíssem óbitos por acidentes de trabalho. Entretanto, há evidências de que o sub-registro de acidentes de trabalho fatais no SINAN é elevado, especialmente entre os trabalhadores informais 14, o que limitaria o seu uso, como também o sistema de informação da Previdência Social pela sua baixa cobertura dos trabalhadores da agropecuária. Isso leva a crer que estimativas precisas requerem pesquisas complementares e o uso de algoritmos de ajustes, como é feito em alguns países 1,5. A falta e insuficiência de dados para os trabalhadores da agropecuária se estende a outras fontes de informação, como a Pesquisa Nacional por Amostra de Domicílios, ou a Pesquisa Mensal de Emprego e Desemprego que não cobrem suficientemente áreas rurais, apesar da existência do Censo Agropecuário realizado a cada 10 anos. Inconsistências foram encontradas entre os dados do censo demográfico e agropecuário para a população economicamente ativa ocupada da agropecuária. Por fim, estudos com dados secundários provenientes de diferentes fontes são um desafio, mas informações de âmbito nacional são necessárias e difíceis de serem obtidas apenas com dados de inquéritos.

Apesar das limitações, esses achados avançam o conhecimento epidemiológico para um amplo segmento de trabalhadores brasileiros, ainda invisível do ponto de vista de estatísticas de agravos relacionados ao trabalho. E exemplifica o uso da recuperação de casos realizada com base em deduções lógicas, valendo-se de dados existentes para os mesmos indivíduos, que permitiu extensa recuperação de registros. Esse procedimento permitiu a identificação de nexo ocupacional presumido de casos com informações faltantes, cujo detalhamento permite o julgamento da pertinência das decisões tomadas pelos investigadores, e da sua reprodução em outros contextos. Isso pode contribuir para a redução do crônico sub-registro de dados sobre acidentes de trabalho fatais, podendo esta estratégia ser empregada em outros estudos, e em especial na vigilância em saúde.

Desigualdades de gênero para intoxicações fatais por agrotóxicos precisam ser melhor investigadas, mas de imediato pode-se recomendar medidas visando a melhor proteção de mulheres trabalhadoras na agropecuária. A informação sobre modos seguros de manusear animais de transporte ou veículos de tração animal, além de equipamentos e implementos agrícolas, precisa ser mais amplamente divulgada empregando-se recursos audiovisuais, visando ao maior impacto. Toda ação em saúde do trabalhador é intersetorial e interdisciplinar, e isto é essencial ao se tratar deste importante ramo de atividade econômica e os seus mais de dez milhões de trabalhadores. 


\section{Colaboradores}

F. N. Ferreira-de-Sousa participou da concepção, extração, tratamento e análise de dados, bem como da elaboração do manuscrito. V. S. Santana participou da concepção, tratamento e análise de dados, e elaboração do manuscrito.

\section{Agradecimentos}

Este trabalho é baseado na dissertação de Mestrado da primeira autora, Programa de Pós-graduação em Saúde Coletiva, Instituto de Saúde Coletiva da Universidade Federal da Bahia (bolsa de Mestrado da Capes). A segunda autora é bolsista de produtividade do $\mathrm{CNPq}$ (processo no 304108/2011-1). É também parte das atividades do Centro Colaborador Vigilância em Saúde do Trabalhador, PISAT/Coordenação Geral da Saúde do Trabalhador, Secretaria de Vigilância em Saúde, Ministério da Saúde.

\section{Referências}

1. Bureau of Labor Statistics. Number and rate of fatal occupational injuries, by industry sector, 2013. Washington DC: United States Department of Labor; 2013.

2. Lee SJ, Kim I, Ryou H, Lee KS, Kwon YJ. Work-related injuries and fatalities among farmers in South Korea. Am J Ind Med 2012; 26:76-83.

3. Mora AM, Mora-Mora MG, Partanen T, Wesseling C. Registration of fatal occupational injuries in Costa Rica, 2005-2006. Int J Occup Environ Health 2011; 17:243-50.

4. Ho S, Wang L, Ho C, Yang C. Fatal occupational injuries in Taiwan, 1994-2005. Occup Environ Med 2010; 67:251-5.
5. European Commission. Eurostat Pocketbooks: Europe social statistics. Luxembourg: Eurostat; 2013.

6. Bureau of Labor Statistics. Number and rate of fatal occupational injuries, by industry sector, 2003. Washington DC: United States Department of Labor; 2003.

7. Varakina ZhL, Vyazmin AN, Sannikov AL, Nygard $\mathrm{CH}$, Grjibovski AN. Fatal occupational injuries in the Arkhangelsk region, Northwest Russia. Occup Med (Lond) 2010; 60:470-5.

8. Dimich-Ward H, Guernsey JR, Pickett W, Rennie D, Hartling L, Brison RJ. Gender differences in the occurrence of farm related injuries. Occup Environ Med 2004; 61:1109-18. 
9. Briere J, Chevalier A, Imbernom E. Surveillance of fatal occupational injuries in France: 2002-2004. Am J Ind Med 2010; 53:1109-18.

10. Thelin A. Fatal accidents in Swedish farming and forestry, 1988-1997. Safety Science 2002; 40: 501-17.

11. Instituto Brasileiro de Geografia e Estatística. Trabalho e rendimento - Censo demográfico 2010. Rio de Janeiro: Instituto Brasileiro de Geografia e Estatística; 2012.

12. Secretaria de Segurança e Saúde no Trabalho, Ministério do Trabalho e Emprego. Norma Regulamentadora no 4. Brasília: Secretaria de Segurança e Saúde no Trabalho, Ministério do Trabalho e Emprego; 1996.

13. Santana VS, Nobre LC, Waldvogel BC. Acidentes de trabalho no Brasil entre 1994 e 2004: uma revisão. Ciênc Saúde Coletiva 2005; 10:841-55.

14. Centro Colaborador da Vigilância em Saúde do Trabalhador. Boletim epidemiológico de acidentes de trabalho fatais. Salvador: Universidade Federal da Bahia/Ministério da Saúde; 2011.

15. Pignati WA, Machado JMH. O agronegócio e seus impactos na saúde dos trabalhadores e da população do Estado de Mato Grosso. In: Gomez CM, Machado JMH, Pena PGL, organizadores. Saúde do trabalhador na sociedade brasileira contemporânea. Rio de Janeiro: Editora Fiocruz; 2011. p. 245-72.

16. Pan American Health Organization; World Health Organization. Health situation in the Americas indicators 20 years - basic indicators. Washington DC: Pan American Health Association/ World Health Organization; 2014.

17. Pan American Health Association; World Health Organization. Regional health observatory. http:// www.paho.org/hq/index.php?option=com_conte nt\&view=article\&id=4535\&Itemid=2391\&lang=en (acessado em 12/Jan/2013).

18. Nobre L, Carvalho FM, Kato M. Validade e acurácia da causa básica na declaração de óbitos por acidentes de trabalho e outras violências. Cad Saúde Colet (Rio J.) 2010; 18:81-93.
19. Corrêa PRL, Assunção AA. A subnotificação de mortes por acidentes de trabalho: estudo de três bancos de dados. Epidemiol Serv Saúde 2003; 12:203-12.

20. Santana VS, Dias EC, Oliveira GL, Moura MCP, Nobre LCC, Machado JMH. Accidentes de trabajo fatales y violencia interpersonal en Brasil, 20002010. Salud Colectiva 2013; 9:139-49.

21. McKnight PE, McKnight KM, Sidani S, Figueiredo AJ. Missing data. New York: The Guilford Press; 2007.

22. Gauterio LW, Bagolin IP. Caracterização dos estabelecimentos agropecuários da Região Sul do Brasil com base no Censo Agropecuário 2006. Revista Economia \& Tecnologia 2011; 7:69-77.

23. Carrington K, Hogg R, McIntosh A, Scott J. ARC Research Project - safeguarding rural Australia : data report no 5 . Brisbane: Centre for Law and Justice; 2009.

24. Coordenação Geral de Informações e Análise Epidemiológica, Secretaria de Vigilância em Saúde, Ministério da Saúde. Sistema de Informações sobre Mortalidade - SIM, consolidação da base de dados de 2011. Brasília: Ministério da Saúde; 2013.

25. Departamento de Análise da Situação em Saúde, Secretaria da Vigilância em Saúde, Ministério da Saúde. Saúde Brasil, 2012. Brasília: Editora do Ministério da Saúde; 2013.

26. Santana VS, Peres MC, Ferreira-de-Sousa FN. Mortalidade por intoxicação ocupacional relacionada a agrotóxicos, 2000-2009, Brasil. Rev Saúde Pública 2013; 47:598-606.

27. Patel SK, Varma MR, Kumar A. Agricultural injuries in Etawah district of Uttar Pradesh in India. Safety Science 2010; 48:222-9.

28. Waggoner JK, Kullman GJ, Henneberger PK, Umbach DM, Blair A, Alavanja MCR, et al. Mortality in the Agricultural Health Study, 1993-2007. Am J Epidemiol 2011; 173:71-83. 


\section{Abstract}

This study estimated annual mortality from work-related injuries in agriculture in Brazil, 2000-2010. The Mortality Information System (SIM) was used to identify cases. Missing data for occupation and work-related injuries were retrieved through other available individual records and incorporated into total cases. Population data were obtained from the official censuses. A total of 8,923 deaths from work-related injuries were identified, of which $44.8 \%$ were located by data retrieval. In the year 2000, estimated crude mortality from work-related injuries was 6.4/100,000 workers, increasing to 8.1/100,000 in 2003 and declining to 7.3/100,000 in 2010. The leading circumstances of deaths in men involved "riding animals or using animal-drawn vehicles", whereas pesticide poisoning was the leading cause in women. Overall mortality from work-related injuries in agriculture was low when compared to that of other countries, suggesting residual under-recording despite data retrieval and thus calling for quality improvement in records. Gender-sensitive preventive measures are necessary.

Rural Workers; Occupational Mortality; Occupational Accidents; Occupational Injuries; Rural Area

\section{Resumen}

Se estima la mortalidad anual por accidentes de trabajo en el sector agropecuario, desde el año 2000 al 2010, en Brasil. Se empleó el Sistema de Información sobre Mortalidad (SIM) para identificar los casos. Los datos ausentes sobre ocupación y accidentes de trabajo se recuperaron con la información individual disponible y se incorporaron al total. Los datos de la población son los de los censos demográficos. Se identificaron 8.923 óbitos por accidentes de trabajo, siendo un $44,8 \%$ de los mismos resultados del proceso de recuperación. En el 2000, la mortalidad bruta por accidentes de trabajo fue estimada en 6,4/100 mil trabajadores, aumentando en 8,1/100 mil en 2003, cuando descendió hasta 7,3/100 mil en 2010. Entre los hombres, "los accidentes con animales o vehículos de tracción animal" fueron las circunstancias más comunes, pero en mujeres fueron las intoxicaciones por pesticidas. La mortalidad general por accidentes de trabajo en el sector agropecuario fue baja, cuando se compara con la de otros países, sugiriendo un sub-registro residual a pesar de la recuperación, lo que requiere una mejoría de la calidad de los registros. Son necesarias medidas de prevención sensibles a las diferencias de género.

Trabajadores Rurales; Mortalidad Laboral; Accidentes de Trabajo; Traumatismos Ocupacionales; Medio Rural
Recebido em 09/Mai/2014

Versão final reapresentada em 09/Jul/2015

Aprovado em 24/Jul/2015 